\title{
CAPACIDADE DE PARASITISMO DE Trichogramma exiguum Pinto \& Platner, 1978 (Hymenoptera: Trichogrammatidae) CRIADO EM DOIS HOSPEDEIROS POR DIVERSAS GERAÇÕES
}

\author{
Parasitism capacity of Trichogramma exiguum Pinto \& Platner, 1978 \\ (Hymenoptera: Trichogrammatidae) reared in two hosts for several generations
}

\author{
Harley Nonato de Oliveira ${ }^{1}$, Carlos Alexandre Colombi ${ }^{2}$, Dirceu Pratissoli ${ }^{2}$, \\ Eder Pin Pedruzzi ${ }^{2}$, Leandro Pin Dalvi ${ }^{2}$
}

\begin{abstract}
RESUMO
O Trichogramma é um inimigo natural dos mais estudados e utilizados atualmente no mundo. A qualidade e o desempenho de Trichogramma podem ser influenciados por alguns fatores, tal como o hospedeiro alternativo utilizado na criação massal. Objetivou-se com este trabalho foi obter informações básicas sobre os aspectos biológicos de Trichogramma exiguum Pinto \& Platner, 1983 (Hymenoptera: Trichogrammatidae), criado nos hospedeiros alternativos Anagasta kuehniella (Zeller, 1879) (Lepidoptera: Pyralidae) e Sitotroga cerealella (Oliv., 1819) (Lepidoptera: Gelechiidae) por 10, 20 e 30 gerações, e os possíveis efeitos nas características biológicas desse parasitóide. Em cada uma das gerações avaliadas, foram utilizadas 25 fêmeas de $T$. exiguum recém-emergidas de cada hospedeiro, as quais foram individualizadas em tubos de vidro que continham gotículas de mel de abelha. Cada fêmea recebeu uma cartela contendo 40 ovos do hospedeiro na qual estava sendo mantido. Foi permitido um parasitismo de 24 horas para essas cartelas, sendo as mesmas trocadas diariamente até a morte da fêmea. O número de ovos parasitados no primeiro dia, a capacidade de parasitismo e a longevidade de T. exiguum foi superior quando se utilizou ovos de A. kuehniella, ao longo das diferentes gerações, mostrando ser esse hospedeiro mais indicado para a criação massal de T. exiguum.
\end{abstract}

TERMOS PARA INDEXAÇÃO: Parasitóide, Anagasta kuehniella, Sitotroga cerealella.

\begin{abstract}
Trichogramma is one the most studied and used natural enemies of insect pests in the world. The quality and the performance of Trichogramma can be influenced by some factors, like the kind of host used for its mass rearing. The objective of this research was to obtain some basic informations about the biological aspects of Trichogramma exiguum Pinto \& Platner, 1983 (Hymenoptera: Trichogrammatidae), reared on Anagasta kuehniella (Zeller, 1879) (Lepidoptera: Pyralidae) and Sitotroga cerealella (Oliv., 1819) (Lepidoptera: Gelechiidae) for 10, 20 e 30 generations, and the effect of something on the biological characteristics of this parasitoid. In each generation, 25 recently emerged females of $T$. exiguum from each host were used, these females being placed individually in glass tubes. Each female received a card with 40 eggs of the host in which it was being maintained. Egg parasitism was allowed for $24 \mathrm{~h}$, with the cards being replaced daily until the death of the parasitoid. The number of eggs parasited in the first day, the capacity of parasitism and the longevity of $T$. exiguum was higher when reared in eggs of A. kuehniella, during the different generations, showing that this host is better indicated for mass rearing of T. exiguum.
\end{abstract}

INDEX TERMS: Parasitoid, Anagasta kuehniella, Sitotroga cerealella.

(Recebido para publicação em 23 de janeiro de 2004 e aprovado em 26 de agosto de 2004)

\section{INTRODUÇÃO}

Espécies do gênero Trichogramma (Hymenoptera: Trichogrammatidae) constituem-se em um dos grupos de inimigos naturais mais estudados e utilizados atualmente no mundo. Os insetos desse gênero são microhimenópteros que parasitam ovos, apresentando ampla distribuição geográfica e um grande número de hospedeiros (ZUCCHI \& MONTEIRO, 1997).
Das 160 espécies do gênero Trichogramma conhecidas em todo mundo (ZUCCHI \& MONTEIRO, 1997), apenas 18 são criadas massalmente em cerca de 16 países e utilizados em liberações inundativas em 18 milhões de hectares (HASSAN, 1997). No Brasil, são grandes as perspectivas de sucesso com o uso de Trichogramma para o controle de pragas, em vista da diversidade de cultivos com potencial de implementação de programas de controle biológico e o grande número de espécies descritas (ZUCCHI \& MONTEIRO, 1997).

\footnotetext{
1. Laboratório de Entomologia - Departamento de Fitotecnia - Centro de Ciências Agrárias da Universidade Federal do Espírito Santo - CCA/UFES Alto Universitário s/n - Alegre, ES - 29500-000 - Bolsista PROFIX do CNPq-Brasil - hnoliveira@insecta.ufv.br

2. Laboratório de Entomologia - Departamento de Fitotecnia - Centro de Ciências Agrárias da Universidade Federal do Espírito Santo - CCA/UFES.
} 
No Brasil, estão registradas 25 espécies distribuídas em quase todas as regiões. É o país da América do Sul com maior número de espécies do gênero Trichogramma conhecidas, associadas a hospedeiros de importância econômica (QUERINO \& ZUCCHI, 2003). No entanto, entre essas espécies não estava registrada Trichogramma exiguum Pinto \& Platner, 1983 (Hymenoptera: Trichogrammatidae), que foi coletada em plantios de tomateiro em Piaçu, distrito de Muniz Freire, ES (OLIVEIRA et al., 2003).

O desempenho de Trichogramma pode ser influenciado por alguns fatores, como hospedeiro alternativo utilizado na criação massal, temperatura, quantidade liberada, área de busca e de químicos que interferem no comportamento de busca (GOODENOUGH \& WITZ, 1985; WAJNBERG \& HASSAN, 1994). Outro fator que pode influenciar esse desempenho é a qualidade desse parasitóide quando criado massalmente, pois esse é um dos requisitos fundamentais para que as características originais do inseto sejam mantidas na criação destes em laboratório (PARRA, 1994).

Objetivou-se com este trabalho obter informações básicas sobre os aspectos biológicos de $T$. exiguum criado nos hospedeiros alternativos Anagasta kuehniella (Zeller, 1879) (Lepidoptera: Pyralidae) e Sitotroga cerealella (Oliv., 1819) (Lepidoptera: Gelechiidae) por 10, 20 e 30 gerações e os possíveis efeitos das mesmas nas características biológicas desse parasitóide.

\section{MATERIAL E MÉTODOS}

O experimento foi conduzido no laboratório de Entomologia do Centro de Ciências Agrárias da Universidade Federal do Espírito Santo (CCA-UFES), em Alegre, ES, em câmara climatizada com temperatura de $25 \pm 1^{\circ} \mathrm{C}$, umidade relativa de $70 \pm 10 \%$ e fotofase de 14 horas.

Foi utilizada uma linhagem de $T$. exiguum coletada em ovos de A. kuehniella, em plantios comerciais de tomate no município de Piaçú, ES. Essa linhagem foi mantida em laboratório por 10, 20 e 30 gerações em ovos de A. kuehniella e S. cerealella.

\section{Criação de Anagasta kuehniella}

A metodologia empregada na criação do hospedeiro alternativo A. kuehniella foi desenvolvida por Parra et al. (1989), porém, foi adaptada para as condições do laboratório de Entomologia do CCA-UFES, utilizando-se uma dieta à base de farinha de trigo integral e de milho (97\%) e levedura de cerveja (3\%).

\section{Criação de Sitotroga cerealella}

O preparo da dieta para a criação do hospedeiro $S$. cerealella teve como base a metodologia desenvolvida por Hassan (1997), porém, foram feitas algumas modificações, utilizando-se uma dieta à base de grãos de trigo descarificado, com umidade variável entre 1114\%. As condições climáticas da sala de criação de A. kuehniella e $S$. cerealella foi de $25 \pm 3^{\circ} \mathrm{C}$ e umidade relativa $70 \pm 10 \%$.

\section{Criação e multiplicação de Trichogramma exiguum}

A criação e multiplicação do parasitóide T. exiguum baseou-se na metodologia descrita por Parra (1997), em que foram oferecidos ovos de S. cerealella e A. kuehniella, sendo os ovos de A. kuehniella, colados em retângulos de cartolina azul celeste $(4,0 \times 2,0 \mathrm{~cm})$ com goma arábica diluída a 10\%. Estas cartelas foram inseridas em recipientes de vidro $(8,5 \times 2,4 \mathrm{~cm})$ contendo adultos recém-emergidos, sendo permitido o parasitismo por 24 horas. As cartelas contendo os ovos parasitados foram mantidas em câmaras climatizadas com temperatura de $25 \pm 1^{\circ} \mathrm{C}$, umidade relativa de $70 \pm 10 \%$ e fotofase de 14 horas.

\section{Capacidade de parasitismo}

Avaliou-se a capacidade de parasitismo de $T$. exiguum na $10^{\mathrm{a}}, 20^{\mathrm{a}}$ e $30^{\mathrm{a}}$ geração de laboratório, criados nos hospedeiros A. kuehniella e S. cerealella.

Em cada uma das gerações avaliadas foram utilizadas 25 fêmeas recém-emergidas de cada hospedeiro, as quais foram individualizadas em tubos de vidro (4,0 $\mathrm{X}$ 0,7cm) contendo gotículas de mel de abelha e tamponados com filme plástico PVC.

Cada fêmea recebeu uma cartela $(3,5 \times$ X 0,5cm) de cor azul-celeste contendo 40 ovos do hospedeiro na qual estava sendo mantida, que foram colados com goma arábica diluída a $10 \%$, sendo os ovos de A. kuehniella inviabilizados em lâmpada germicida por 50 minutos. Foi permitido um parasitismo de 24 horas para essas cartelas, sendo as mesmas trocadas diariamente até a morte da fêmea. Após esse período de parasitismo, as cartelas foram acondicionadas em tubos de vidro (8,5 X $2,4 \mathrm{~cm}$ ) e mantidas nas mesmas condições de criação do parasitóide até a emergência dos descendentes e contagem dos ovos parasitados. 


\section{RESULTADOS E DISCUSSÃO}

O maior número de ovos parasitados por $T$. exiguum foi registrado no primeiro dia de parasitismo, em ambos hospedeiros utilizados, e em todas as gerações (Gráfico 1). Resultados semelhantes também foram relatados por Oliveira et al. (2003), quando utilizou $T$. exiguum em ovos de Corcyra cephalonica (Stainton, 1865) (Lepidoptera: Pyralidae), por Pinto \& Tavares (1991), que também encontraram maior parasitismo de Trichogramma cordubensis Vargas e Cabello, 1985 (Hymenoptera: Trichogrammatidae) em ovos de $A$. kuehniella, no primeiro dia, assim como Pratissoli \& Oliveira (1999), que também observaram maior parasitismo de Trichogramma pretiosum Riley, 1879 (Hymenoptera: Trichogrammatidae) no primeiro dia em ovos de Helicoverpa zea (Boddie, 1850) (Lepidoptera: Noctuidae).

Os maiores valores observados para a capacidade de parasitismo de T. exiguum, foram quando este parasitóide foi criado em ovos de A. kuehniella, enquanto os menores valores foram observados quando $T$. exiguum foi mantido em ovos de $S$. cerealella, indiferente das gerações avaliadas (Gráfico 1). Resende \& Ciociolla (1996) relataram uma capacidade de parasitismo para Trichogramma atopovirilia Oatman e Platner, 1983 (Hymenoptera: Trichogrammatidae) de 45,2 ovos/fêmea quando parasitaram ovos de $H$. zea. Tironi (1992) encontrou para esse mesmo parasitóide uma capacidade de parasitismo de 57 ovos/fêmea quando criado no hospedeiro A. kuehniella. Sá (1991) obteve em média 51 ovos/fêmea para T. pretiosum em ovos de H. zea. Basso et al. (1998) relatou que houve uma variação de 38,5 a 60,5 ovos/fêmea na capacidade de parasitismo de diferentes linhagens de T. exiguum parasitando ovos de $A$. kuehniella. Esses dados revelam que a capacidade de parasitismo de Trichogramma pode variar em função do hospedeiro alternativo no qual ele foi criado, das espécies de parasitóide pertencentes a esse gênero e até mesmo em uma mesma espécie em função de suas linhagens.

A longevidade, nas diferentes gerações, variou de 11 a 13 dias para fêmeas de T. exiguum, advindas de ovos de A. kuehniella e de 6 a 10 dias em ovos de $S$. cerealella (Gráfico 1). Lewis et al. (1976) também encontraram maior longevidade em ovos de A. kuehniella do que em ovos de $S$. cerealella para Trichogramma sp. Berti \& Marcano (1993), encontraram longevidade de $T$. pretiosum, em ovos de $S$. cerealella, similar a encontrada nesse trabalho, e Bai et al. (1995), mostraram que para Trichogramma brassicae Bezdenko, 1968, Trichogramma minutum Riley, 1879 e Trichogramma nr. sibiricum Sorokina, 1984 (Hymenoptera: Trichogrammatidae), ocorre variação na longevidade entre diferentes espécies (8,6 a 9,2 dias, de 10,2 a 11,7 e de 8,3 a 12,4 respectivamente). Os resultados relatados por diferentes autores, mostram que a longevidade pode variar em função do hospedeiro e da espécie de Trichogramma que esta sendo criada.

Os resultados encontrados mostraram que o número de ovos parasitados no primeiro dia, a capacidade de parasitismo e a longevidade de T. exiguum foi superior quando se utilizou como hospedeiro alternativo, ovos de A. kuehniella. $10^{\mathrm{a}}$ Geração de Trichogramma exiguum em ovos de Anagasta kuehniella

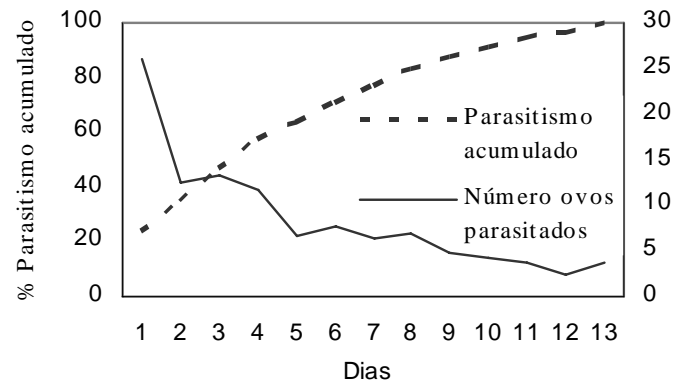

$10^{\mathrm{a}}$ Geração de Trichogramma exiguum em ovos de Sitotroga cerealella

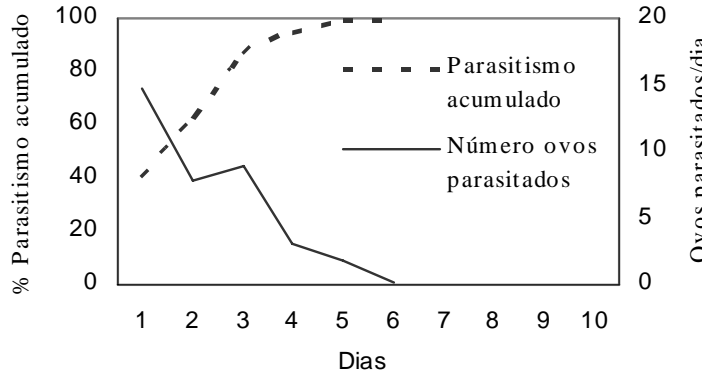

Continua...

Ciênc. agrotec., Lavras, v. 29, n. 2, p. 284-288, mar./abr., 2005 
Continuação...

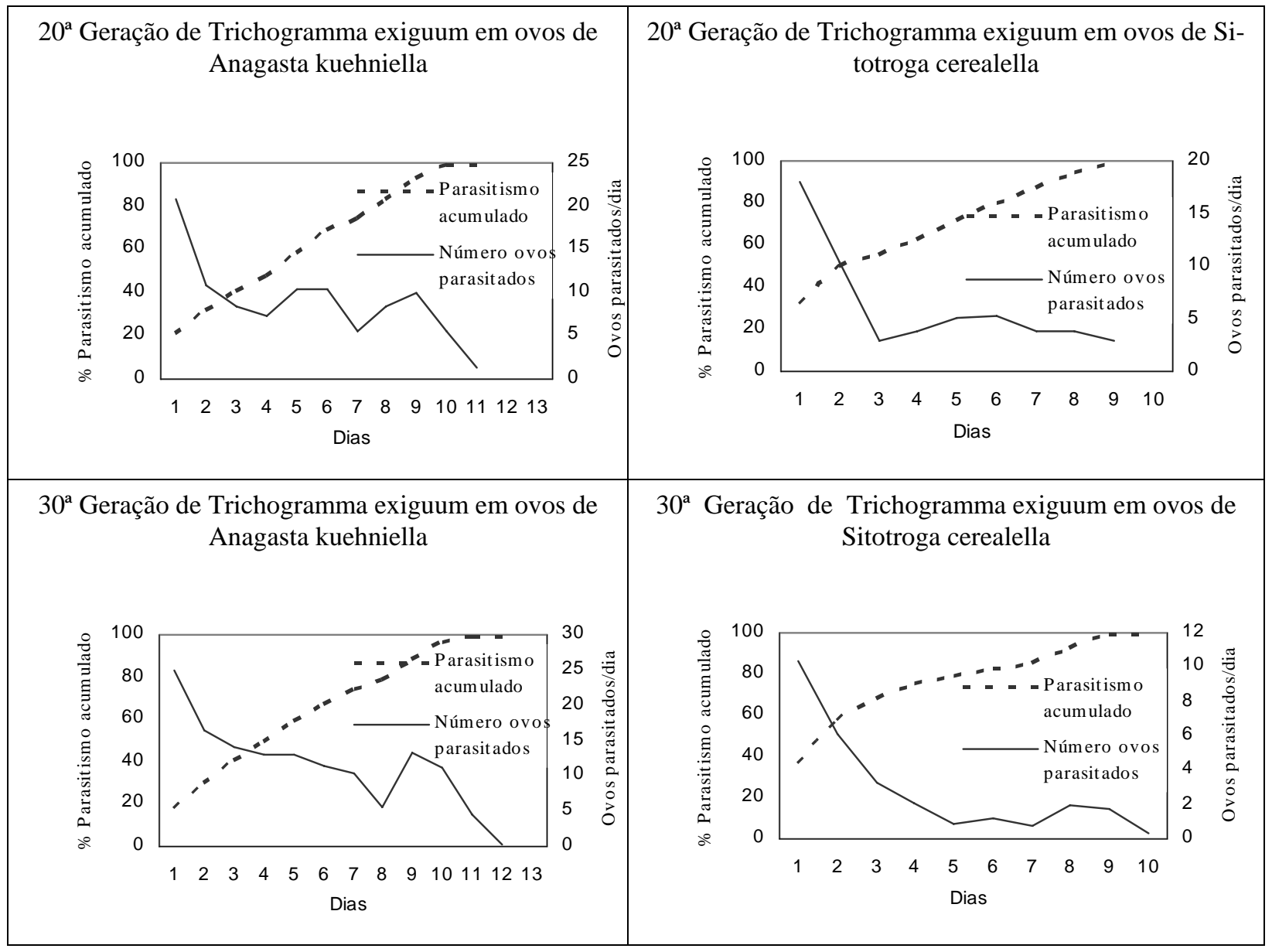

GRÁFICO 1 - Porcentagem de parasitismo acumulado e número de ovos parasitados/dia de Trichogramma exiguum, na $10^{\mathrm{a}}$, $20^{\mathrm{a}}$ e $30^{\mathrm{a}}$ geração, em ovos de Anagasta kuehniella e Sitotroga cerealella.

\section{CONCLUSÃO}

Conclui-se que o hospedeiro A. kuehniella é mais indicado que $S$. cerealella para a criação massal de $T$. exiguum, pois com esse hospedeiro, o parasitóide apresenta maior capacidade de parasitismo e menor variação das características biológicas avaliadas, ao longo das diversas gerações.

\section{AGRADECIMENTOS}

Ao CNPq (Conselho Nacional de Desenvolvimento Científico e Tecnológico) pelas bolsas e auxílio concedidos.

\section{REFERÊNCIAS BIBLIOGRÁFICAS}

BAI, B.; ÇOBANOGLU, S.; SMITH, S. M. Assessment of Trichogramma species for biological control of forest lepidopteran defoliators. Entomologia experimentalis et applicata, Dordrecht, v. 75, p. 135-145, 1995.

BASSO, C.; GRILLE, G.; POMPANON, F.; ALLEMAND, R.; PINTUREAU, B. Comparacion de los caracteres biológicos y etológicos de Trichogramma pretiosum y de T. exiguum (Hymenoptera: Trichogrammatidae). Revista Chilena de Entomologia, Santiago, v. 25, p. 45-53, 1998. 
BERTI, J.; MARCANO, R. Effect of different food substances on the reproduction and lifespan of the female of Trichogramma pretiosum Riley (Hymenoptera: Trichogrammatidae). Boletin de Entomologia Venezolana, Maracay, v. 8, n. 2, p. 105-110, 1993.

HASSAN, S. A. Criação da traça do milho, Sitotroga cerealella, para a produção massal de Trichogramma. In: PARRA, J. R. P.; ZUCCHI, R. A. (Eds.). Trichogramma e o controle biológico aplicado. Piracicaba: FEALQ, 1997. p. 173-182, 324 p.

LEWIS, W. J.; NORDLUND, D. A.; GROSS JUNIOR, H. R.; PERKINS, W. D.; KNIPLING, E. F.; VOEGELE, J. Production and performance of Trichogramma reared on eggs of Heliothis zea and other hosts. Environmental Entomology Lanham, College Park, v. 5, n. 3, p. 449-452, 1976.

GOODENOUGH, J. L.; WITZ, J. A. Modeling augmentative releases of Trichogramma pretiosum. The Southwestern Entomologist, College Station, v. 8, p. 169-189, 1985.

OLIVEIRA, H. N.; PRATISSOLI, D.; COLOMBI, C. A.; ESPINDULA, M. C. Características biológicas de Trichogramma exiguum Pinto \& Platner em ovos de Corcyra cephalonica Stainton. Magistra, Salvador, v. 15, n. 1, p. 103-105, 2003.

PARRA, J. R. P. Técnicas de criação de insetos para programas de controle biológico. Piracicaba: FEALQ, 1994. $192 \mathrm{p}$.

PARRA, J. R. P. Técnicas de criação de Anagasta kuehniella, hospedeiro alternativo para produçào de Trichogramma. In: PARRA, J. R. P.; ZUCCHI, R. A. Trichogramma e o controle biológico aplicado. Piracicaba: FEALQ, 1997. p. 121-150.

PARRA, J. R. P.; LOPES, J. R. S.; SERRA, H. J. P. Metodologia de criação de Anagasta kuehniella (Zeller, 1879) para produção massal de Trichogramma spp. Anais Sociedade Entomologia Brasileira, Londrina, v. 18, n. 2, p. 403-415, 1989.

PINTO, F.; TAVARES, J. Longevity and parasitic capacity of the Azorean type of Trichogramma cordubensis Vargas; Cabello (Hymenoptera: Trichogrammatidae). In: INTERNATIONAL SYMPOSIUM ON TRICHOGRAMMA AND OTHER EGG PARASITOIDS, 3., 1990, San Antonio. Proceeding... Paris: INRA, 1991. p. 89-91.

PRATISSOLI, D.; OLIVEIRA, H. N. Influência da idade do ovos de Helicoverpa zea (Boddie) no parasitismo de Trichogramma pretiosum Riley. Pesquisa Agropecuária Brasileira, Brasília, v. 34, n. 5, p. 891-896, 1999.

QUERINO, R. B.; ZUCCHI, R. A. O gênero Trichogramma (Hymenoptera:Trichogrammatidae) no Brasil. In: SIMPÓSIO DE CONTROLE BIOLÓGICO, 8., 2003, São Pedro. Resumos... São Paulo: SICONBIOL, 2003. p. 131.

RESENDE, D. L. M. C.; CIOCIOLLA, A. I. Capacidade de parasitismo de Trichogramma atopovirilia Oatman \& Platner, 1983 (Hymenoptera: Trichogrammatidae) em ovos de Helicoverpa zea (Boddie, 1850) (Lepidoptera: Noctuidae) em diferentes temperaturas. Ciência e Agrotecnologia, Lavras, v. 20, n. 4, p. 421-424, 1996.

SÁ, L. A. N. de. Bioecologia de Trichogramma pretiosum Riley, 1879, visando avaliar seu potencial para controle de Spodoptera frugiperda (J.E. Smith, 1797) e Helicoverpa zea (Boddie, 1850) em milho. 1991. 107 f. Tese (Doutorado em Entomologia) - Escola Superior de Agricultura Luiz de Queiroz, Piracicaba, 1991.

TIRONI, P. Aspectos bioecológicos de Trichogramma pretiosum Riley, 1879 e Trichogramma atopovirilia Oatman \& Platner, 1983 (Hymenoptera: Trichogrammatidae), como agentes de controle bioecológico de Helicoverpa zea (Boddie, 1850) (Lepidoptera: Noctuidae) em milho. 1992. 74 f. Dissertação (Mestrado em Entomologia) - Escola Superior de Agricultura de Lavras, Lavras, 1992.

WAJNBERG, E.; HASSAN, S. A. Biological control with egg parasitoids. Wallingford: British Library, 1994. 286 p.

ZUCCHI, R. A.; MONTEIRO, R. C. O gênero Trichogramma na América do Sul. In: PARRA, J. R. P.; ZUCCHI, R. A. (Eds.). Trichogramma e o Controle Biológico Aplicado. Piracicaba: FEALQ, 1997. p. 41-66. 